\title{
Characterization of the Murine K14-FynY528F Transgenic SCC Model Provides Novel Therapeutic Insights
}

\section{Michael D Gober and John T Seykora*}

Perelman School of Medicine at the University of Pennsylvania, Department of Dermatology Biomedical Research Building, Philadelphia PA 19104, USA

\begin{abstract}
Cutaneous squamous cell carcinoma (cSCC) is one of the most common human malignancies. The development of mouse models that accurately recapitulate the human condition is crucial for testing novel therapeutic modalities. In this article we discuss the utility of the K14-Fyn-Y528F transgenic mouse as a model for cSCC and actinic keratosislike precursor lesions and its use in testing novel small molecule kinase inhibitors topically.
\end{abstract}

Keywords: Cutaneous squamous cell carcinoma; Malignancy; Mutations; Neoplasia

\section{Introduction and Rationale}

Cutaneous squamous cell carcinoma (cSCC), the second most common malignancy in the US, has reached epidemic proportions with over 250,000 new lesions per year [1].The precursor lesion for cSCC, termed actinic keratosis (AK), is associated with UV exposure and frequently located in skin adjacent to cSCC [1]. While the rate of progression from AK to Cscc can be difficult to measure, it is estimated to range between $0.25 \%$ and $16 \%$ per year for an individual lesion $[2,3]$. The typical patient over 40 years old with sun damaged skin will have six to eight individual AKs at any given time [4,5]. The significance of the epidemic of cSCCs was highlighted by the Surgeon General in a recent 'Call to Action' regarding UV-induced skin cancers which called for further research regarding these lesions [6]. Given the large overall numbers of individuals affected by AKs and CSCC, generation and use of physiologically relevant models is of paramount importance for the development of neweffective therapeutic modalities.

The classic multistep model for carcinogenesis is useful for understanding the progression of AKs to cutaneous malignancy [7]. In this model, mutations in one gene, typically a tumor suppressor such as p53, lead to genomic instability and loss of cell cycle control which is followed by additional mutations in oncogenes promoting neoplasia. Indeed, p53 is commonly mutated in AK and SCC in situ (cSCC is) suggesting that the loss of p53 activity is an early event in cutaneous carcinogenesis [8]. This is in contrast to many internal malignancies in which p53 mutation/loss of function is a late finding associated with invasive phenotype [9-11]. The multistep hypothesis is complicated by the fact that oncogenes can regulate p53 activity $[12,13]$. Organotypic 3D models of cutaneous carcinogenesis have shown that activating mutations in two oncogenes is sufficient to drive cSCC formation [14$16]$.

Increased Src-family kinase (SFK) activity has been identified both in human AKs and cSCCs[13]. Increased activation of Fyn, an SFK, leads to downregulation of p53 transcript and protein demonstrating that signaling mechanisms can downregulate p53 independent of gene mutation[13]. Fyn activation has been associated with increased proliferation, invasion and metastasis and is well known, like other SFKs,to activate the pro-oncogenic Ras/MEK/ERK proliferation pathway [17-19]. The ability of activated Fyn to downregulate antioncogenes involved in cutaneous carcinogenesis, such as p53, as well as drive the pro-oncogenic Ras-MAP kinase pathway suggests that a transgenic mouse expressing constitutively activated Fyn will be a good model for both AK and cSCC.

\section{Generation and Characterization of the K14-FYN- Y528F Model}

The conventional murine cutaneous carcinogenesis model involves exposing the mice to two chemicals designed to induce mutations that lead to SCC-like tumors. In this "two-step" model of cSCC-like lesions are induced after exposing the mouse skin to a tumor initiator such as 7,12-dimethylbenz[a]anthracene (DMBA) followed by 12-O-tetradecanoylphorbol-13-acetate (TPA) as the tumor promoter [20]. The precursor lesion in this chemical carcinogenesis model is a precancerous papilloma which architecturally and histologically does not resemble a human $\mathrm{AK}$ or $\mathrm{CSCC}$ is. The inablitity to generate precursor lesions that mimic human SCC precursor lesions is a potential drawback when using this model to test candidate novel therapeutics designed to target precursor lesions. To generate a mouse model of $\mathrm{AK}$ and $\mathrm{CSCC}$ that better mimics the human disease, our laboratory developeda transgenic mouse that expressed an activated form of Fyn (Fyn-Y528F) in the epidermis. To drive Fyn Y528F expression in the epidermis, we generated akeratin 14 (K14) Fyn transgene which would be expressed in the basal layer of the epidermis; the strategy is widely used to preferentially express transgenes in the epidermis. The effect of the K14-Fyn Y528F transgeneon tumor formation was first evaluated in C57BL/6 mice, a tumor resistant genetic background [13].The C57/BL6 K14-Fyn Y528F transgenic mice developed hyperkeratotic plaques within 3-4 days and approximately $21 \%$ went on to develop keratotic tumors by 3 to 7 months of age that were cSCCs[13]. Figure 1 shows a reprehensive C57/BL6 K14-Fyn Y528F transgenic mouse with spontaneous SCC-like tumors on the back and associated histologic and biomarker analysis that is consistent with SCC. In order to achieve a model with more tumors and a shorter latency period for tumor development the K14-Fyn-Y528F construct was crossed into the FVB/ Nmouse line, a more tumor-permissive genetic background. The FVB/N K14-Fyn Y528Fmice spontaneously form tumors at 5 to 8 weeks of agewith a histologic appearance of well-differentiated cSCC. They

*Corresponding author: John T. Seykora,Associate Professor, Departments of Dermatology and Pathology, Member CAMB Graduate Group, Member Abramson Cancer Center, Perelman School of Medicine at the University of Pennsylvania, Room 1011 BRB II/III, 421 Curie Blvd.Philadelphia, PA 19104, USA, Tel: 215-8980170; Fax: 215-573-2143; E-mail: john.seykora@uphs.upenn.edu

Received January 22, 2015; Accepted April 09, 2015; Published April 11, 2015

Citation: Gober MD, Seykora JT (2015) Characterization of the Murine K14FynY528F Transgenic SCC Model Provides Novel Therapeutic Insights. Med chem 5: 149-151. doi:10.4172/2161-0444.1000256

Copyright: ( 2015 Gober MD, et al. This is an open-access article distributed under the terms of the Creative Commons Attribution License, which permits unrestricted use, distribution, and reproduction in any medium, provided the original author and source are credited. 


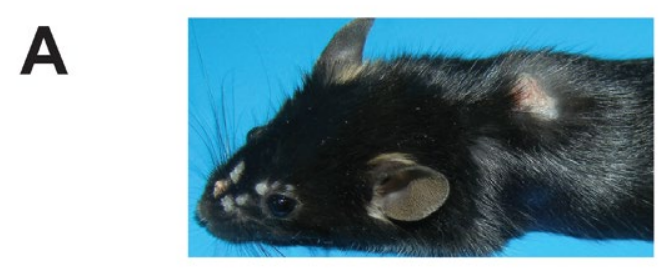

B
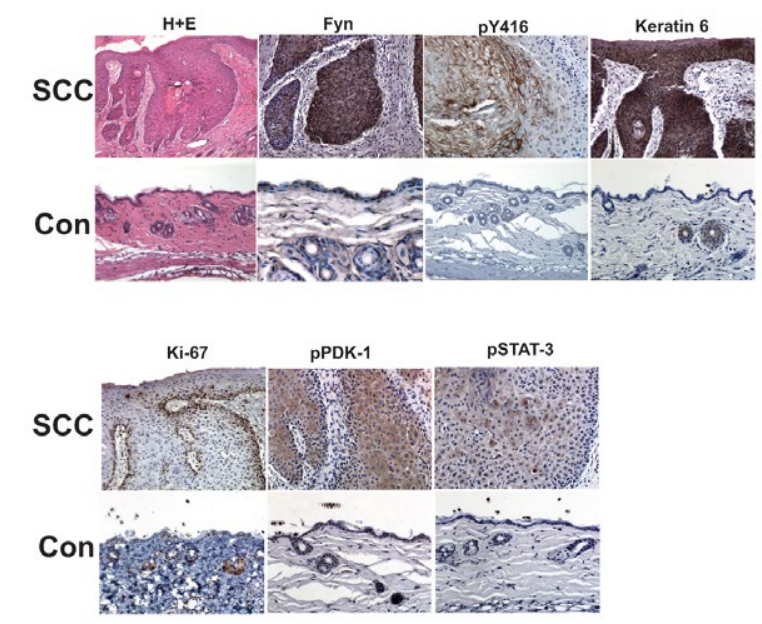

Figure 1: K14-Fyn-Y528F mice exhibits both AK-like and SCC lesions. (A) A representative transgenic mouse demonstrating many hyperkeratotic tumors on the back, ears, and face (yellow arrows). (B) Formalin fixed, paraffin embedded mouse skin section stained by H\&E followed by immunohistochemical and biomarker analysis.

also develop flat hyperkeratotic lesions consistent with precancerous lesions that histologically resemble cSCC in situ (cSCCis) and actinic keratosis (AK), respectively [13]. The incidence of hyperkeratotic plaques resembling AKs and SCCis was $81 \%$ and spontaneous CSCC formation was seen in $36 \%$ of mice [13].

The Fyn-Y528F transgene led to down regulation of p53 and Notch1similar to the inactivation of p53 and Notch1 seen in human cSCCs[13,21].Increased oncogenic signaling was observed in the K14-FynY528F tumors including activation of Erk, Stat-3, and Pdk-1mirroring what has been seen in human cSCCs and CSCC is. Furthermore, these mice did not develop metastatic cSCC when followed for up to 6 months mimicking the low metastatic potential observed with most human cSCCs[13]. Collectively, these data support the conclusion that K14-Fyn-Y528 transgenic mice accurately model human AKs and cSCCs. The benefit of a physiologically relevant in vivo model is that it represents a pre-clinical screening tool to test potential novel therapeutics for human CSCC and associated precursor lesions with regard to both safety and efficacy prior to human trials. One potential caveat when using the K14-Fyn Y528F model is the inherent differences between mouse and human skin. Therapeutics that may show promise in this mouse model may not possess the same therapeutic efficacy when tested in humans.

Currently, 5-fluorouracil (5-FU)and imiquimod are FDA approved for the topical treatment of AKs. 5-FU is a chemotherapeutic agent that broadly inhibits DNA and RNA synthesis in dividing cells while imiquimod is a TLR7 agonist that promotes the elimination of AKs through stimulating the local immune response [22,23]. These therapeutic agents are often associated with skin inflammation and irritation leading to morbidity and poor adherence to the treatment regimen[22].

\section{Experimental Applications of the K14-FYN-Y528F Model}

The K14-Fyn-Y528F mice model can serve as a means to test the efficacy of newly targeted therapies as compared to conventional topical therapy. Lesions in this transgenic mouse model occur rapidly after birth facilitating rapid testing of candidate compounds. Since the signaling pathways activated in these mice mirror the pathways activated in human AKs and cSCCs, small molecule kinase inhibitors targeting the signaling pathways can be evaluated for efficacy and safety in this model. Currently kinase inhibitors such as dasatinib are used systemically to treat invasive head and neck squamous cell carcinoma [24]. Since many small molecule inhibitors are small enough to penetrate the skin they theoretically can be used topically to treat AK or cutaneous SCCis lesions. Studies are currently underway using the K14-Fyn- Y528F mouse model testing topical general tyrosine kinase inhibitors as well as kinase inhibitors of the PI3 kinase/AKT pathway and the MEK/ERK pathway. Our preliminary results indicate that when applied once daily in a gel formulation at concentrations ranging from $0.5 \%$ to $1.5 \%$ (corresponding to $10 \mathrm{mM}$ to $30 \mathrm{mM}$ drug concentration for a drug that is approximately $500 \mathrm{Da}$ ) topical kinase inhibitors induced tumor shrinkage without associated inflammation or ulceration. Since this mouse was developed in our laboratory, it is available directly from us at the University of Pennsylvania. Alternatively we have made this mouse available through Jackson Laboratory (http://www.jax. org) under the strain name FVB.Cg-Tg(KRT14-Fyn ${ }^{\star}$ )aJsey/J (stock \# 017005). We anticipate that the widespread use of the K14-Fyn-Y528 mouse model will greatly enhance the pace of discovery for novel therapeutics to treat AK, cSCCis and CSCC.

\section{Acknowledgements}

We would like to thank National Institute of Arthritis and Musculoskeletal and SkinDiseases grant RO1-AR051380 andR01-CA165836, U Penn SDRC grant 5-P30-AR057217, Dermatology Foundation, Skin Cancer Foundation and the Department of Dermatology, University of Pennsylvania Medical School.

\section{References}

1. Rogers HW, Weinstock MA, Harris AR, Hinckley MR, Feldman SR, et al. (2010) Incidence estimate of nonmelanoma skin cancer in the United States, 2006. Arch Dermatol 146: 283-287.

2. Glogau RG (2000) The risk of progression to invasive disease. J Am Acad Dermatol 42: 23-24.

3. Marks R, Rennie G, Selwood TS (1988) Malignant transformation of solar keratoses to squamous cell carcinoma. Lancet 1: 795-797.

4. Marks R (1995) An overview of skin cancers. Incidence and causation. Cancer 75: 607-612.

5. Mittelbronn MA, Mullins DL, Ramos-Caro FA, Flowers FP (1998) Frequency of pre-existing actinic keratosis in cutaneous squamous cell carcinoma. Int $J$ Dermatol 37: 677-681.

6. U.S. Department of Health and Human Services (2014)The Surgeon General's Call to Action to Prevent Skin Cancer.Washington, DC. U.S. Dept of Health and Human Services Office of the Surgeon General.

7. Ratushny V, Gober MD, Hick R, Ridky TW, Seykora JT (2012) From keratinocyte to cancer: the pathogenesis and modeling of cutaneous squamous cell carcinoma. J Clin Invest 122: 464-472.

8. Ortonne JP (2002) From actinic keratosis to squamous cell carcinoma. $\mathrm{Br} \mathrm{J}$ Dermatol 146 Suppl 61: 20-23.

9. Baker SJ, Preisinger AC, Jessup JM, Paraskeva C, Markowitz S, et al. (1990) p53 gene mutations occur in combination with $17 \mathrm{p}$ allelic deletions as late events in colorectal tumorigenesis. Cancer Res 50: 7717-7722.

10. Donghi R, Longoni A, Pilotti S, Michieli P, Della Porta G, et al. (1993) Gene p53 mutations are restricted to poorly differentiated and undifferentiated carcinomas of the thyroid gland. J Clin Invest 91: 1753-1760.

11. Uchida T, Wada C, Ishida H, Wang C, Egawa S, et al. (1995) p53 mutations and prognosis in bladder tumors. J Urol 153: 1097-1104. 
Citation: Gober MD, Seykora JT (2015) Characterization of the Murine K14-FynY528F Transgenic SCC Model Provides Novel Therapeutic Insights. Med chem 5: 149-151. doi:10.4172/2161-0444.1000256

12. Kolev V, Mandinova A, Guinea-Viniegra J, Hu B, Lefort K, et al. (2008) EGFR signalling as a negative regulator of Notch1 gene transcription and function in proliferating keratinocytes and cancer. Nat Cell Biol 10: 902-911.

13. Zhao L, Li W, Marshall C, Griffin T, Hanson M, et al. (2009) Srcasm inhibits Fyn-induced cutaneous carcinogenesis with modulation of Notch1 and p53. Cancer Res 69: 9439-9447.

14. Dajee M, Lazarov M, Zhang JY, Cai T, Green CL, et al. (2003) NF-kappaB blockade and oncogenic Ras trigger invasive human epidermal neoplasia. Nature 421: 639-643.

15. Lazarov M, Kubo Y, Cai T, Dajee M, Tarutani M, et al. (2002) CDK4 coexpression with Ras generates malignant human epidermal tumorigenesis. Nat Med 8: 1105-1114.

16. Ridky TW, Chow JM, Wong DJ, Khavari PA (2010) Invasive three-dimensional organotypicneoplasia from multiple normal human epithelia. Nat Med 16: 14501455.

17. Abe J, Berk BC (1999) Fyn and JAK2 mediate Ras activation by reactive oxygen species. J Biol Chem 274: 21003-21010.

18. Yadav V, Denning MF (2011) Fyn is induced by Ras/PI3K/Akt signaling and is required for enhanced invasion/migration. MolCarcinog 50: 346-352.
19. Ayli EE, Li W, Brown TT, Witkiewicz A, Elenitsas R, et al. (2008) Activation of Src-family tyrosine kinases in hyperproliferative epidermal disorders. J Cutan Pathol 35: 273-277.

20. Gober MD, Bashir HM, Seykora JT (2013) Reconstructing skin cancers using animal models. Cancer Metastasis Rev 32: 123-128.

21. South AP, Purdie KJ, Watt SA,Haldenby $S$, den Breems NY, et al. (2014) NOTCH1 mutations occur early during cutaneous squamous cell carcinogenesis. J Invest Dermatol 134: 2630-2638.

22. Weinstock MA, Lee KC, Chren MM, Marcolivio K; VATTC Trial Group (2009) Quality of life in the actinic neoplasia syndrome: The VA Topical Tretinoin Chemoprevention (VATTC) Trial. J Am AcadDermatol 61: 207-215.

23. Berman B, Amini S (2012) Pharmacotherapy of actinic keratosis: an update Expert Opin Pharmacother 13: 1847-1871.

24. Safdari Y, Khalili M, Farajnia S, Asgharzadeh M, Yazdani Y, et al. (2014) Recent advances in head and neck squamous cell carcinoma--a review. Clin Biochem 47: 1195-1202. 